TRANSACTIONS OF THE

AMERICAN MATHEMATICAL SOCIETY

Volume 353, Number 11, Pages 4481-4496

S 0002-9947(01)02791-X

Article electronically published on May 9, 2001

\title{
HOMOTOPY COMMUTATIVITY OF $H$-SPACES WITH FINITELY GENERATED COHOMOLOGY
}

\author{
YUSUKE KAWAMOTO AND JAMES P. LIN
}

\begin{abstract}
We show that a simply connected homotopy associative and homotopy commutative mod $3 \mathrm{H}$-space with finitely generated mod 3 cohomology is homotopy equivalent to a finite product of $K(\mathbb{Z}, 2), S p(2)$, the threeconnected cover $S p(2)\langle 3\rangle$ and the homotopy fiber $S p(2)\left\langle 3 ; 3^{i}\right\rangle$ of the map $\left[3^{i}\right]: S p(2) \rightarrow K(\mathbb{Z}, 3)$ for $i \geq 1$. Our result also shows that a connected $C_{p}$-space in the sense of Sugawara with finitely generated mod $p$ cohomology has the homotopy type of a finite product of $K(\mathbb{Z}, 1), K(\mathbb{Z}, 2)$ and $K\left(\mathbb{Z} / p^{i}, 1\right)$ for $i \geq 1$.
\end{abstract}

\section{INTRODUCTION}

$H$-space theory is a homotopical generalization of group theory, and it is one of the major objects in unstable homotopy theory. In recent years, many theorems have been proved about finite $H$-spaces (cf. [12],[15]), which suggest that they are similar to compact Lie groups. In this paper, we study $H$-spaces which need not be finite, but whose mod $p$ cohomology rings are finitely generated. One should note that the three-connected cover of a simply connected compact Lie group is such an $H$-space. Other examples are Eilenberg-Mac Lane spaces $K(\mathbb{Z}, 1), K(\mathbb{Z}, 2)$ and $K\left(\mathbb{Z} / p^{i}, 1\right)$ for $i \geq 1$. At present, only a few results are known for such $H-$ spaces (cf. [5],[8], [13], [14], [16], 22], 23]). Recently, Broto and Crespo [5], [8] gave an important result for $H$-spaces with finitely generated cohomology. They have shown that such an $H$-space is the total space of an $H$-fibration over a finite $H$ space. Their result can be used to reduce problems about $H$-spaces with finitely generated cohomology to ones of finite $H$-spaces.

Let $p$ be a prime. Throughout this paper, we assume that all spaces are completed at $p$ in the sense of Bousfield-Kan [4, and the cohomology rings are taken with $\mathbb{Z} / p$-coefficients unless otherwise specified. An $H$-space which is completed at $p$ is called a mod $p H$-space. In this paper, we study the homotopy commutativity of mod $p H$-spaces with finitely generated $\bmod p$ cohomology.

In the case of $p=2$, Slack proved the following classification theorem:

Theorem 1.1 ([22, Cor. 0.2], [5, Cor. 1.5]). If $X$ is a connected homotopy commutative mod $2 \mathrm{H}$-space such that the mod 2 cohomology $H^{*}(X)$ is finitely generated as an algebra, then $X$ is homotopy equivalent to a finite product of EilenbergMac Lane spaces $K(\mathbb{Z}, 1), K(\mathbb{Z}, 2)$ and $K\left(\mathbb{Z} / 2^{i}, 1\right)$ for $i \geq 1$.

Received by the editors February 12, 1999 and, in revised form, September 18, 2000

2000 Mathematics Subject Classification. Primary 55P45, 55P15; Secondary 55P65.

Key words and phrases. $H$-space, $C_{n}$-space, Dror Farjoun localization functor.

Partially supported by JSPS Research Fellowships for Young Scientists. 
Broto-Crespo 5 reproved the above theorem by using another method. At odd primes, it is known that any connected $\bmod p H$-space admits an $H$-structure which is homotopy commutative by a result of Iriye-Kono [10. It is not known, however, what are the necessary conditions for an $H$-space to have its $H$-structure which is homotopy associative and homotopy commutative at the same time.

In the case of $p=3$, we give a classification of homotopy associative and homotopy commutative mod $3 \mathrm{H}$-spaces with finitely generated mod 3 cohomology. It is known that the compact Lie group $S p(2)$ and the three-connected cover $S p(2)\langle 3\rangle$ are such $H$-spaces (see [18 Thm. 2]). If $S p(2)\left\langle 3 ; 3^{i}\right\rangle$ denotes the homotopy fiber of the map of degree $3^{i}$ :

$$
\left[3^{i}\right]: S p(2) \longrightarrow K(\mathbb{Z}, 3),
$$

then we see that $S p(2)\left\langle 3 ; 3^{i}\right\rangle$ is also a homotopy associative and homotopy commutative mod $3 \mathrm{H}$-space with finitely generated mod 3 cohomology for $i \geq 1$.

Our first result is stated as follows:

Theorem A. If $X$ is a simply connected homotopy associative and homotopy commutative mod $3 \mathrm{H}$-space such that the mod 3 cohomology $H^{*}(X)$ is finitely generated as an algebra, then $X$ is homotopy equivalent to a finite product of $K(\mathbb{Z}, 2)$, $S p(2), S p(2)\langle 3\rangle$ and $S p(2)\left\langle 3 ; 3^{i}\right\rangle$ for $i \geq 1$.

Slack [23] proved that for an odd prime $p$, if $X$ is a connected homotopy associative and homotopy commutative $\bmod p H$-space with finitely generated $\bmod p$ cohomology, then the even degree generators of $H^{*}(X)$ are concentrated in degrees $2 p^{i}$ for $i \geq 0$. As a corollary of Theorem $\mathrm{A}$, we can generalize his result in the case of $p=3$.

Corollary 1.2. If $X$ is a connected homotopy associative and homotopy commutative mod $3 H$-space such that the mod 3 cohomology $H^{*}(X)$ is finitely generated as an algebra, then the even degree generators of $H^{*}(X)$ are concentrated in degrees 2 and 18.

To describe an odd prime version of Theorem 1.1, we need to generalize the homotopy commutativity of $H$-spaces to the higher ones. Sugawara [25] introduced a notion of the higher homotopy commutativity of loop spaces. He used it to give a criterion of a homotopy commutative loop space to be the loop space of an $H-$ space. If $X$ is a loop space, then by using the loop structure in the sense of Moore, we have a multiplication $\mu: X \times X \rightarrow X$ on $X$ which is strictly associative. We call $X$ a $C_{n}$-space if the multiplication $\mu: X \times X \rightarrow X$ is an $A_{n}-$ map in the sense of Stasheff [24] and Sugawara [25]. We see that $X$ is a $C_{2}$-space if and only if $X$ is a homotopy commutative loop space, and by Sugawara [25, Thm. 4.2], $X$ is a $C_{\infty}$-space if and only if $X$ is the loop space of an $H$-space.

Our second result is stated as follows:

Theorem B. Let $p$ be an odd prime. If $X$ is a connected $C_{p}$-space such that the $\bmod p$ cohomology $H^{*}(X)$ is finitely generated as an algebra, then $X$ is homotopy equivalent to a finite product of Eilenberg-Mac Lane spaces $K(\mathbb{Z}, 1), K(\mathbb{Z}, 2)$ and $K\left(\mathbb{Z} / p^{i}, 1\right)$ for $i \geq 1$.

McGibbon [19, Thm. 3] proved that if $X$ is a connected finite $C_{p}$-space, then $X$ has the homotopy type of a torus. Theorem B generalizes his result to the case of $C_{p}$-spaces with finitely generated $\bmod p$ cohomology. We see that Theorem B 
also generalizes results of Lin [14] and Kawamoto [13], in which the same result was proved under the assumption that $X$ is the loop space of an $H$-space.

This paper is organized as follows: In $\S 2$ we recall the Dror Farjoun localization functor with respect to a space introduced in [9]. It is shown that the localization functor preserves several homotopical structures on $H$-spaces (see Proposition 2.3 and Proposition 2.5). In $\S 3$ we prove Theorem A and Corollary 1.2. By combining Proposition 2.3 with results of Broto and Crespo [5], [8], we can reduce Theorem A to the case of mod 3 finite $H$-spaces. From a result of Lin [17, we can determine the homotopy type of a homotopy associative and homotopy commutative mod 3 finite $H$-space (see Proposition 3.1). $\S 4$ is devoted to the proof of Theorem B. By using Proposition 2.5 and a result of McGibbon 19, we complete the proof of Theorem B. We also give a necessary condition for a $\bmod p$ loop space with finitely generated $\bmod p$ cohomology to be a $C_{p-1}$-space (see Theorem 4.5).

This research was done while the first author was visiting the University of California, San Diego. He would like to express his hearty thanks to Professor J.P. Lin for his hospitality. The authors would like to thank Professor A. Kono for his useful comments to the first draft of this paper in which we had shown Theorem A on the cohomological level. His opinion was very helpful for us to improve Theorem A. We are also grateful to Professor T. Matumoto, Professor Y. Hemmi and Professor M. Imaoka for their valuable suggestions. Finally, we appreciate the referee for many useful comments.

\section{Dror FARJoun LOCALIZATION FUnCTOR}

Dror Farjoun 9 introduced the localization functor with respect to a space. We show that the localization functor preserves several homotopical structures on $H$ spaces. In Proposition 2.3, it is shown that the localization functor preserves the homotopy associativity and the homotopy commutativity of $H$-spaces. In Proposition 2.5, we treat the higher homotopy commutativity of loop spaces. These results are used in $\S 3$ and $\S 4$.

First we recall the localization functor. Let $A$ be a space. A space $X$ is called $A$-local if the base point evaluation map $\epsilon: \operatorname{Map}(A, X) \rightarrow X$ is a homotopy equivalence, or equivalently, if the pointed mapping space $\operatorname{Map}_{*}(A, X)$ is contractible. Let $\mathcal{S}$ denote the category of spaces having the homotopy type of $C W$-complexes. By Dror Farjoun [9] Thm. 1.A.3], there is a homotopy functor $L_{A}: \mathcal{S} \rightarrow \mathcal{S}$ with respect to $A$. We call $L_{A}$ the $A$-localization functor. Given a space $X$, the $A$-localization $L_{A}(X)$ is $A$-local, and we have the natural map $\psi_{X}: X \rightarrow L_{A}(X)$.

It is shown in 9 , that $L_{A}$ has several properties similar to those of the $p$ completion functor $(\mathbb{Z} / p)_{\infty}$ of Bousfield-Kan [4]. One of them is that the natural map $\psi_{X}$ is homotopically universal to $A$-local spaces. Given an $A$-local space $W$ and a map $\zeta: X \rightarrow W$, there exists an extension $\tilde{\zeta}: L_{A}(X) \rightarrow W$ unique up to homotopy with $\tilde{\zeta} \psi_{X} \simeq \zeta$. Furthermore, it is known by [9, p.18] that $\psi_{X}$ induces a homotopy equivalence

$$
\left(\psi_{X}\right)^{*}: \operatorname{Map}\left(L_{A}(X), W\right) \longrightarrow \operatorname{Map}(X, W)
$$

for an $A$-local space $W$. Dror Farjoun [9, Thm. 1.C.13] proved that $L_{A}$ is a continuous homotopy functor, that is, the map

$$
\lambda_{A}: \operatorname{Map}(X, Y) \longrightarrow \operatorname{Map}\left(L_{A}(X), L_{A}(Y)\right)
$$


defined by $\lambda_{A}(g)=L_{A}(g)$ is continuous when the compact-open topology is assigned to these mapping spaces. We note that (2.1) also implies the continuity of $L_{A}$ since $\lambda_{A}$ can be represented by $\lambda_{A}=\omega\left(\psi_{Y}\right)_{*}$, where $\omega: \operatorname{Map}\left(X, L_{A}(Y)\right) \rightarrow$ $\operatorname{Map}\left(L_{A}(X), L_{A}(Y)\right)$ denotes the homotopy inverse of $\left(\psi_{X}\right)^{*}$. The continuity of the $p$-completion functor $(\mathbb{Z} / p)_{\infty}$ is proved by Iwase [11, Thm. 3.7]. He used it to show that $(\mathbb{Z} / p)_{\infty}$ strictly preserves the higher homotopy associativity of $H$-spaces. We also use the continuity to show that $L_{A}$ preserves several homotopical structures on $H$-spaces. But we need more precise arguments since $L_{A}$ is in general a homotopy functor (see Proposition 2.3).

It is known by [4, Prop. VI 6.5] that the $p$-completion functor $(\mathbb{Z} / p)_{\infty}$ always preserves a fibration. But the $A$-localization functor $L_{A}$ does not necessarily preserve a fibration. Dror Farjoun proved the following result:

Proposition 2.1 ([9] Thm. 1.H.1, Cor. 3.D.3]). Let $F \rightarrow E \rightarrow X$ be a fibration, where $F, E$ and $X$ are connected spaces. If $L_{A}(F)$ is contractible or $X$ is $A$-local, then the localization $L_{A}$ preserves the fibration.

Given a space $X$, let $F_{A}(X)$ denote the composite $(\mathbb{Z} / p)_{\infty}\left(L_{A}(X)\right)$. By composing the natural maps $\psi_{X}: X \rightarrow L_{A}(X)$ and $L_{A}(X) \rightarrow(\mathbb{Z} / p)_{\infty}\left(L_{A}(X)\right)$, we have the natural map $\phi_{X}: X \rightarrow F_{A}(X)$.

In the proof of Proposition 2.3 and Proposition 2.5, we need the following lemma:

Lemma 2.2. Let $A$ be a space. If $X$ is a space, then the natural map $\psi_{X}: X \rightarrow$ $L_{A}(X)$ induces homotopy equivalences

$$
\begin{aligned}
& \left(\left(\psi_{X}\right)^{k}\right)^{*}: \operatorname{Map}\left(L_{A}(X)^{k}, L_{A}(X)\right) \longrightarrow \operatorname{Map}\left(X^{k}, L_{A}(X)\right), \\
& \left(\left(\psi_{X}\right)^{k}\right)^{*}: \operatorname{Map}_{*}\left(L_{A}(X)^{k}, L_{A}(X)\right) \longrightarrow \operatorname{Map}_{*}\left(X^{k}, L_{A}(X)\right)
\end{aligned}
$$

for $k \geq 1$.

Proof. By a result of Dror Farjoun [9, 1.A.8 e.4], there exists a homotopy equivalence $\xi_{X^{k}, X}: L_{A}\left(X^{k+1}\right) \rightarrow L_{A}\left(X^{k}\right) \times L_{A}(X)$ with $\xi_{X^{k}, X} \psi_{X^{k+1}} \simeq \psi_{X^{k}} \times \psi_{X}$ for $k \geq 1$. If we define a map $\xi_{k}: L_{A}\left(X^{k}\right) \rightarrow L_{A}(X)^{k}$ by $\xi_{k}=\left(\xi_{X, X} \times\right.$

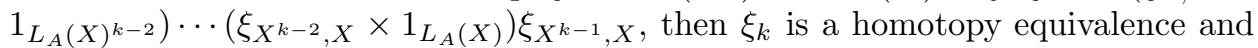
$\left(\psi_{X}\right)^{k} \simeq \xi_{k} \psi_{X^{k}}$. By $(2.1)$, we see that

$$
\left(\psi_{X^{k}}\right)^{*}: \operatorname{Map}\left(L_{A}\left(X^{k}\right), L_{A}(X)\right) \rightarrow \operatorname{Map}\left(X^{k}, L_{A}(X)\right)
$$

is a homotopy equivalence, which implies that $\left(\left(\psi_{X}\right)^{k}\right)^{*}: \operatorname{Map}\left(L_{A}(X)^{k}, L_{A}(X)\right) \rightarrow$ $\operatorname{Map}\left(X^{k}, L_{A}(X)\right)$ is also a homotopy equivalence.

If we consider the following homotopy commutative diagram of fibrations:

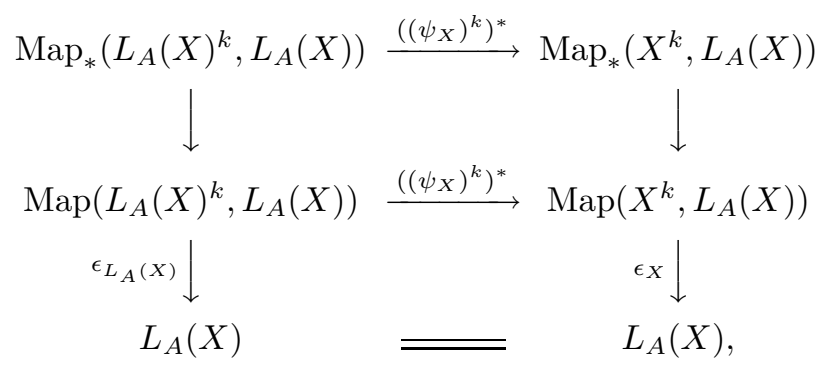

then the top horizontal arrow is also a homotopy equivalence, where $\epsilon_{X}$ and $\epsilon_{L_{A}(X)}$ denote the base point evaluation maps. This completes the proof. 
Now we recall the definitions of an $A_{3}$-map between homotopy associative $H$ spaces and a $C_{2}$-map between homotopy commutative $H$-spaces. Let $X$ and $Y$ be $H$-spaces, and let $\phi: X \rightarrow Y$ be an $H$-map. Then we have a map $F_{2}$ : $X^{2} \times I \rightarrow Y$ such that $F_{2}\left(x_{1}, x_{2}, 0\right)=\phi\left(x_{1}\right) \cdot \phi\left(x_{2}\right)$ and $F_{2}\left(x_{1}, x_{2}, 1\right)=\phi\left(x_{1} \cdot x_{2}\right)$ for $x_{1}, x_{2} \in X$. If $X$ and $Y$ are homotopy associative $H$-spaces, then there exist maps $M_{3}: X^{3} \times I \rightarrow X$ and $N_{3}: Y^{3} \times I \rightarrow Y$ satisfying that

$$
\begin{aligned}
& M_{3}\left(x_{1}, x_{2}, x_{3}, 0\right)=\left(x_{1} \cdot x_{2}\right) \cdot x_{3}, \\
& M_{3}\left(x_{1}, x_{2}, x_{3}, 1\right)=x_{1} \cdot\left(x_{2} \cdot x_{3}\right)
\end{aligned}
$$

and

$$
\begin{aligned}
& N_{3}\left(y_{1}, y_{2}, y_{3}, 0\right)=\left(y_{1} \cdot y_{2}\right) \cdot y_{3} \\
& N_{3}\left(y_{1}, y_{2}, y_{3}, 1\right)=y_{1} \cdot\left(y_{2} \cdot y_{3}\right)
\end{aligned}
$$

respectively. $\phi: X \rightarrow Y$ is called an $A_{3}$-map if there exists a map $F_{3}: X^{3} \times I^{2} \rightarrow Y$ satisfying that

$$
\begin{aligned}
& F_{3}\left(x_{1}, x_{2}, x_{3}, 0, t_{2}\right)= \begin{cases}F_{2}\left(x_{1}, x_{2}, 2 t_{2}\right) \cdot \phi\left(x_{3}\right) & \text { if } 0 \leq t_{2} \leq 1 / 2, \\
F_{2}\left(x_{1} \cdot x_{2}, x_{3}, 2 t_{2}-1\right) & \text { if } 1 / 2 \leq t_{2} \leq 1,\end{cases} \\
& F_{3}\left(x_{1}, x_{2}, x_{3}, 1, t_{2}\right)= \begin{cases}\phi\left(x_{1}\right) \cdot F_{2}\left(x_{2}, x_{3}, 2 t_{2}\right) & \text { if } 0 \leq t_{2} \leq 1 / 2, \\
F_{2}\left(x_{1}, x_{2} \cdot x_{3}, 2 t_{2}-1\right) & \text { if } 1 / 2 \leq t_{2} \leq 1,\end{cases} \\
& F_{3}\left(x_{1}, x_{2}, x_{3}, t_{1}, 0\right)=N_{3}\left(\phi\left(x_{1}\right), \phi\left(x_{2}\right), \phi\left(x_{3}\right), t_{1}\right), \\
& F_{3}\left(x_{1}, x_{2}, x_{3}, t_{1}, 1\right)=\phi\left(M_{3}\left(x_{1}, x_{2}, x_{3}, t_{1}\right)\right)
\end{aligned}
$$

for $x_{1}, x_{2}, x_{3} \in X$.

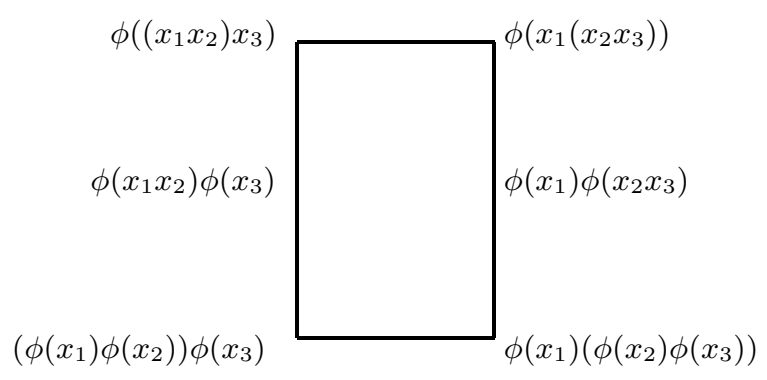

FiguRE 1. $A_{3}$-structure on $\phi$

If we assume that $X$ and $Y$ are homotopy commutative $H$-spaces, then there exist maps $Q_{2}: X^{2} \times I \rightarrow X$ and $R_{2}: Y^{2} \times I \rightarrow Y$ satisfying that

$$
\begin{aligned}
& Q_{2}\left(x_{1}, x_{2}, 0\right)=x_{1} \cdot x_{2}, \\
& Q_{2}\left(x_{1}, x_{2}, 1\right)=x_{2} \cdot x_{1}
\end{aligned}
$$

and

$$
\begin{aligned}
& R_{2}\left(y_{1}, y_{2}, 0\right)=y_{1} \cdot y_{2}, \\
& R_{2}\left(y_{1}, y_{2}, 1\right)=y_{2} \cdot y_{1}
\end{aligned}
$$


respectively. We call $\phi: X \rightarrow Y$ a $C_{2}-$ map if there exists a map $D_{2}: X^{2} \times I^{2} \rightarrow Y$ satisfying that

$$
\begin{aligned}
& D_{2}\left(x_{1}, x_{2}, 0, t_{2}\right)=F_{2}\left(x_{1}, x_{2}, t_{2}\right), \\
& D_{2}\left(x_{1}, x_{2}, 1, t_{2}\right)=F_{2}\left(x_{2}, x_{1}, t_{2}\right), \\
& D_{2}\left(x_{1}, x_{2}, t_{1}, 0\right)=R_{2}\left(\phi\left(x_{1}\right), \phi\left(x_{2}\right), t_{1}\right), \\
& D_{2}\left(x_{1}, x_{2}, t_{1}, 1\right)=\phi\left(Q_{2}\left(x_{1}, x_{2}, t_{1}\right)\right)
\end{aligned}
$$

for $x_{1}, x_{2} \in X$.

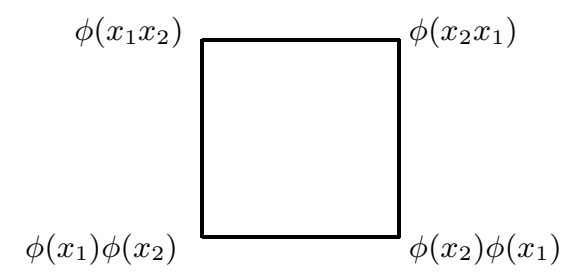

Figure 2. $C_{2}$-structure on $\phi$

In the proof of Theorem A, we need the following result:

Proposition 2.3. Let $A$ be a space. If $X$ is a homotopy associative and homotopy commutative mod $p H$-space, then $F_{A}(X)$ is also a homotopy associative and homotopy commutative mod $p H$-space, and $\phi_{X}: X \rightarrow F_{A}(X)$ is an $A_{3}-m a p$ and a $\mathrm{C}_{2}-$ map at the same time.

We have the following lemma by an elementary argument:

Lemma 2.4. Let $A$ and $B$ be spaces, and let $\phi: A \rightarrow B$ be a homotopy equivalence. If $(K, L)$ is a relative $C W$-complex and there are maps $f: K \rightarrow B$ and $g: L \rightarrow A$ with $\phi g=\left.f\right|_{L}$, then there exists a map $h: K \rightarrow A$ such that $\left.h\right|_{L}=g$ and $\phi h \simeq$ $f$ rel $L$.

Now we prove Proposition 2.3 as follows:

Proof of Proposition 2.3. First we show that $F_{A}(X)$ is a $\bmod p H$-space and $\phi_{X}$ : $X \rightarrow F_{A}(X)$ is an $H$-map. By Dror Farjoun [9, 1.A.8 e.4], there exists a homotopy equivalence $\gamma: L_{A}(X)^{2} \rightarrow L_{A}\left(X^{2}\right)$ with $\gamma\left(\psi_{X}\right)^{2} \simeq \psi_{X^{2}}$. Let $\mu: X^{2} \rightarrow X$ denote the $H$-multiplication on $X$. We define a map $\nu: L_{A}(X)^{2} \rightarrow L_{A}(X)$ by $\nu=L_{A}(\mu) \gamma$. Then $\nu\left(\psi_{X}\right)^{2} \simeq \psi_{X} \mu$ since $L_{A}(\mu) \psi_{X^{2}} \simeq \psi_{X} \mu$. Let $\iota_{j}: L_{A}(X) \rightarrow L_{A}(X)^{2}$ be the inclusion on the $j$-th factor for $j=1,2$. Since $\nu \iota_{j} \psi_{X} \simeq \psi_{X}$, we have by Lemma 2.4 that $\nu \iota_{j} \simeq 1_{L_{A}(X)}$ for $j=1,2$, and so $L_{A}(X)$ is an $H$-space so that $\psi_{X}: X \rightarrow L_{A}(X)$ is an $H$-map. By a result of Iwase [11, Cor 3.10], we have that $F_{A}(X)$ is also a mod $p H$-space and $\phi_{X}: X \rightarrow F_{A}(X)$ is an $H$-map.

Since $X$ is a homotopy associative $H$-space and $\psi_{X}: X \rightarrow L_{A}(X)$ is an $H$-map, there exist maps $M_{3}: X^{3} \times I \rightarrow X$ and $F_{2}: X^{2} \times I \rightarrow L_{A}(X)$ satisfying the above conditions. Let $\sigma: I \rightarrow \operatorname{Map}\left(X^{3}, X\right)$ and $\tau: I \rightarrow \operatorname{Map}\left(X^{2}, L_{A}(X)\right)$ denote the adjoint maps of $M_{3}$ and $F_{2}$, respectively. Then we have that

$$
\begin{aligned}
& \sigma(0)=\mu\left(\mu \times 1_{X}\right), \\
& \sigma(1)=\mu\left(1_{X} \times \mu\right)
\end{aligned}
$$


and

$$
\begin{aligned}
& \tau(0)=\psi_{X} \mu, \\
& \tau(1)=\nu\left(\psi_{X}\right)^{2},
\end{aligned}
$$

and so we can define a map $\kappa: \partial I \times I \cup I \times\{1\} \rightarrow \operatorname{Map}\left(X^{3}, L_{A}(X)\right)$ by

$$
\begin{aligned}
& \kappa\left(0, t_{2}\right)= \begin{cases}\nu\left(\tau\left(2 t_{2}\right) \times \psi_{X}\right) & \text { if } 0 \leq t_{2} \leq 1 / 2, \\
\tau\left(2 t_{2}-1\right)\left(\mu \times 1_{X}\right) & \text { if } 1 / 2 \leq t_{2} \leq 1,\end{cases} \\
& \kappa\left(1, t_{2}\right)= \begin{cases}\nu\left(\psi_{X} \times \tau\left(2 t_{2}\right)\right) & \text { if } 0 \leq t_{2} \leq 1 / 2, \\
\tau\left(2 t_{2}-1\right)\left(1_{X} \times \mu\right), & \text { if } 1 / 2 \leq t_{2} \leq 1\end{cases}
\end{aligned}
$$

and $\kappa\left(t_{1}, 1\right)=\psi_{X}\left(\sigma\left(t_{1}\right)\right)$. From the homotopy extension property, we have a map $\tilde{\kappa}: I^{2} \rightarrow \operatorname{Map}\left(X^{3}, L_{A}(X)\right)$ with $\left.\tilde{\kappa}\right|_{\partial I \times I \cup I \times\{1\}}=\kappa$. Let $\gamma: I \rightarrow \operatorname{Map}\left(X^{3}, L_{A}(X)\right)$ and $\zeta: \partial I \rightarrow \operatorname{Map}\left(L_{A}(X)^{3}, L_{A}(X)\right)$ be the maps defined by $\gamma(t)=\tilde{\kappa}(t, 1), \zeta(0)=$ $\nu\left(\nu \times 1_{L_{A}(X)}\right)$ and $\zeta(1)=\nu\left(1_{L_{A}(X)} \times \nu\right)$, respectively. We see that $\left(\left(\psi_{X}\right)^{3}\right)^{*}(\zeta)=$ $\left.\gamma\right|_{\partial I}$, and so by Lemma 2.2 and Lemma 2.4 , there exists a map

$$
\tilde{\zeta}: I \rightarrow \operatorname{Map}\left(L_{A}(X)^{3}, L_{A}(X)\right)
$$

such that $\left.\tilde{\zeta}\right|_{\partial I}=\zeta$ and $\left(\left(\psi_{X}\right)^{3}\right)^{*}(\tilde{\zeta}) \simeq \gamma$ rel $\partial I$. From the above constructions, we have a map $\omega: I^{2} \rightarrow \operatorname{Map}\left(X^{3}, L_{A}(X)\right)$ such that $\left.\omega\right|_{\partial I \times I \cup I \times\{1\}}=\kappa$ and $\omega\left(t_{1}, 0\right)=$ $\left(\left(\psi_{X}\right)^{3}\right)^{*}\left(\tilde{\zeta}\left(t_{1}\right)\right)$. Let $N_{3}: L_{A}(X)^{3} \times I \rightarrow L_{A}(X)$ and $F_{3}: X^{3} \times I^{2} \rightarrow L_{A}(X)$ denote the adjoint maps of $\tilde{\zeta}$ and $\omega$, respectively. We see that they satisfy the conditions (2.2) and (2.3), and so $L_{A}(X)$ is a homotopy associative $H$-space so that $\psi_{X}: X \rightarrow L_{A}(X)$ is an $A_{3}$-map. By a result of Iwase [11, Cor. 3.10], we can conclude that $F_{A}(X)$ is also a homotopy associative mod $p H$-space and $\phi_{X}: X \rightarrow F_{A}(X)$ is an $A_{3}$-map.

By using similar arguments to the above, we can show that the localization $F_{A}(X)$ is a homotopy commutative $\bmod p H$-space and the natural map $\phi_{X}$ : $X \rightarrow F_{A}(X)$ is a $C_{2}-$ map, and so we have the required conclusion. This completes the proof of Proposition 2.3.

Next we recall the definition of a $C_{n}$-space in the sense of Sugawara [25]. If $X$ is a loop space, then by using the Moore loop structure, we can assume that $X$ is a strictly associative $H$-space. $X$ is said to be a $C_{n}$-space if the multiplication $\mu: X \times X \rightarrow X$ is an $A_{n}$-map in the sense of Sugawara [25] and Stasheff [24, Def. II 4.4], that is, there exist maps $G_{k}: X^{2(k+1)} \times I^{k} \rightarrow X$ for $0 \leq k<n$ satisfying that $G_{0}(x, y)=\mu(x, y)=x \cdot y$,

$$
\begin{aligned}
G_{k}\left(x_{0}, y_{0}, \cdots, x_{k}, y_{k}, t_{1}, \cdots, t_{k}\right) \\
=\left\{\begin{array}{cc}
G_{k-1}\left(x_{0}, x_{1}, \cdots, x_{i-2}, y_{i-2}, x_{i-1} \cdot x_{i}, y_{i-1} \cdot y_{i},\right. \\
\left.x_{i+1}, y_{i+1}, \cdots, x_{k}, y_{k}, t_{1}, \cdots, t_{i-1}, t_{i+1}, \cdots, t_{k}\right) & \text { if } t_{i}=0, \\
G_{i-1}\left(x_{0}, x_{1}, \cdots, x_{i-1}, y_{i-1}, t_{1}, \cdots, t_{i-1}\right) & \text { if } t_{i}=1 \\
\cdot G_{k-i}\left(x_{i}, y_{i} \cdots, x_{k}, y_{k}, t_{i+1}, \cdots, t_{k}\right) &
\end{array}\right.
\end{aligned}
$$

for $1 \leq k<n$ and

$$
G_{k}\left(*, \cdots, *, t_{1}, \cdots, t_{k}\right)=*
$$


for $0 \leq k<n$. From the definition, we see that $X$ is a $C_{2}$-space if and only if $X$ is a homotopy commutative loop space. Figure 3 and Figure 4 denote the $C_{3}$-structure and the $C_{4}$-structure on $X$, respectively. Furthermore, by Sugawara [25. Thm. 4.2], $X$ is a $C_{\infty}$-space if and only if $X$ has the homotopy type of the loop space of an $H$-space.

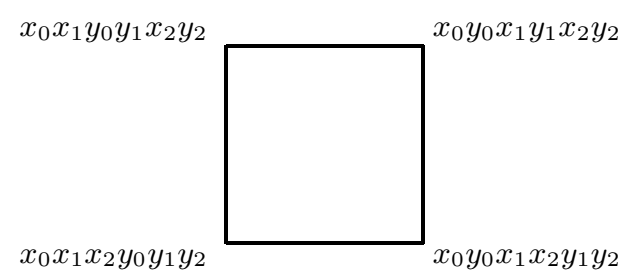

FiguRE $3 . C_{3}$-structure on $X$

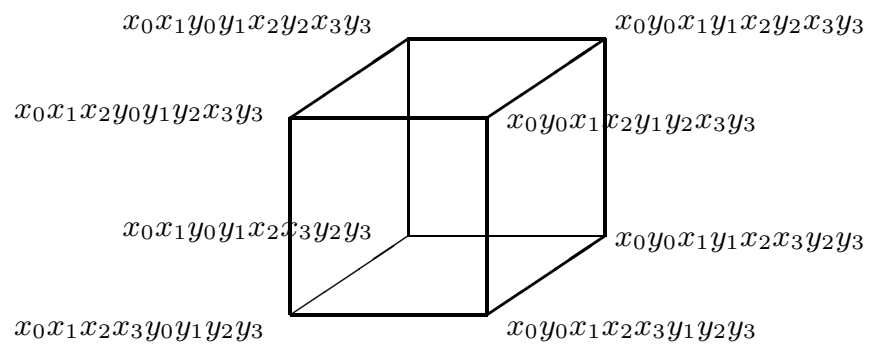

Figure 4. $C_{4}$-structure on $X$

If we assume that $X$ and $Y$ are $C_{n}$-spaces, then there exist maps $G_{k}: X^{2(k+1)} \times$ $I^{k} \rightarrow X$ and $H_{k}: Y^{2(k+1)} \times I^{k} \rightarrow Y$ for $0 \leq k<n$ satisfying the conditions (2.4) and (2.5). A loop map $\phi: X \rightarrow Y$ is said to be a $C_{n}$-map if there exist maps $L_{k}$ : $X^{2(k+1)} \times I^{k+1} \rightarrow Y$ for $0 \leq k<n$ satisfying that $L_{0}(x, y, s)=\phi(x) \cdot \phi(y)=\phi(x \cdot y)$ for $s \in I$,

$$
\begin{aligned}
L_{k}\left(x_{0}, y_{0}, \cdots, x_{k}, y_{k}, t_{1}, \cdots, t_{k}, s\right) \\
\quad= \begin{cases}H_{k}\left(\phi\left(x_{0}\right), \phi\left(y_{0}\right), \cdots, \phi\left(x_{k}\right), \phi\left(y_{k}\right), t_{1}, \cdots, t_{k}\right) & \text { if } s=0, \\
\phi\left(G_{k}\left(x_{0}, y_{0}, \cdots, x_{k}, y_{k}, t_{1}, \cdots, t_{k}\right)\right) & \text { if } s=1,\end{cases}
\end{aligned}
$$

$$
\begin{aligned}
L_{k}( & \left.x_{0}, y_{0}, \cdots, x_{k}, y_{k}, t_{1}, \cdots, t_{k}, s\right) \\
\quad= & \left\{\begin{array}{cc}
L_{k-1}\left(x_{0}, x_{1}, \cdots, x_{i-2}, y_{i-2}, x_{i-1} \cdot x_{i}, y_{i-1} \cdot y_{i},\right. \\
\left.x_{i+1}, y_{i+1}, \cdots, x_{k}, y_{k}, t_{1}, \cdots, t_{i-1}, t_{i+1}, \cdots, t_{k}, s\right) & \text { if } t_{i}=0 \\
L_{i-1}\left(x_{0}, x_{1}, \cdots, x_{i-1}, y_{i-1}, t_{1}, \cdots, t_{i-1}, s\right) & \\
\cdot L_{k-i}\left(x_{i}, y_{i} \cdots, x_{k}, y_{k}, t_{i+1}, \cdots, t_{k}, s\right) & \text { if } t_{i}=1
\end{array}\right.
\end{aligned}
$$

for $1 \leq k<n$ and 


$$
L_{k}\left(*, \cdots, *, t_{1}, \cdots, t_{k}, s\right)=*
$$

for $0 \leq k<n$.

In the proof of Theorem $\mathrm{B}$, we need the following result:

Proposition 2.5. If $X$ is a $C_{n}$-space, then the localization $F_{A}(X)$ is a $C_{n}$-space and the natural map $\phi_{X}: X \rightarrow F_{A}(X)$ is a $C_{n}-$ map.

Proof of Proposition 2.5. We give an outline of the proof since the proof is similar to the one of Proposition 2.3. If $X$ is a loop space, then we have a space $W$ with $X=\Omega W$. By a result of Dror Farjoun [9, Thm. 3.A.1], there is a homotopy equivalence $\zeta: L_{A}(X) \rightarrow \Omega L_{\Sigma A}(W)$ with $\zeta \psi_{X} \simeq \Omega\left(\psi_{W}\right): X \rightarrow \Omega L_{\Sigma A}(W)$. It is sufficient to show that $Y=\Omega L_{\Sigma A}(W)$ is a $C_{n}$-space and $\theta=\Omega\left(\psi_{W}\right): X \rightarrow Y$ is a $C_{n}$-map.

If we consider the following homotopy commutative diagram:

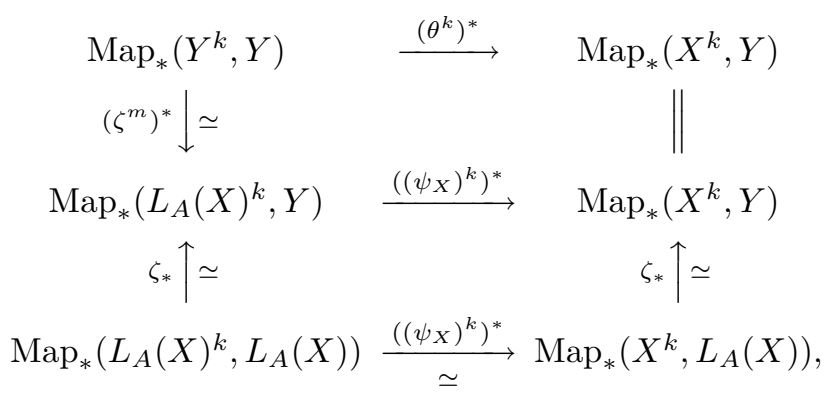

then by Lemma 2.2, the bottom horizontal arrow is a homotopy equivalence, and so we have that

$$
\left(\theta^{k}\right)^{*}: \operatorname{Map}_{*}\left(Y^{k}, Y\right) \longrightarrow \operatorname{Map}_{*}\left(X^{k}, Y\right)
$$

is a homotopy equivalence for $k \geq 1$. By using similar arguments to the proof of Proposition 2.3, and by using Lemma 2.4 and (2.9), we can construct maps $H_{k}: Y^{2(k+1)} \times I^{k} \rightarrow Y$ and $L_{k}: X^{2(k+1)} \times I^{k+1} \rightarrow Y$ for $0 \leq k<n$ satisfying the conditions $(2.4-5)$ and $(2.6-8)$.

Iwase [11, Cor. 3.10] has shown that the $p$-completion functor $(\mathbb{Z} / p)_{\infty}$ preserves the higher homotopy associativity of $H$-spaces. By using the same arguments, we can show that $(\mathbb{Z} / p)_{\infty}$ also preserves a $C_{n}$-structure on loop spaces. Since $F_{A}$ is the composite of the functors $L_{A}$ and $(\mathbb{Z} / p)_{\infty}$, we have the required conclusion. This completes the proof of Proposition 2.5.

\section{Proof of Theorem A}

In this section we prove Theorem A and Corollary 1.2. First we determine the homotopy type of a simply connected homotopy associative and homotopy commutative mod 3 finite $H$-space (see Proposition 3.1). By using Proposition 3.1, Proposition 2.3 and results of Broto and Crespo [5], $[8]$, we can prove Theorem A. Next we complete the proof of Corollary 1.2 by using a spectral sequence argument.

If $X$ is a connected homotopy associative and homotopy commutative $\bmod p$ finite $H$-space, then by a result of Browder [7, Thm. 8.6], the mod $p$ cohomology $H^{*}(X)$ is an exterior algebra generated by the odd dimensional generators. In the case of $p>3$, his result is the best possible. In fact, if $p>3$, then the odd dimensional sphere $S^{2 n-1}$ is a homotopy associative and homotopy commutative $\bmod p H$-space for any $n \geq 1$. 
Recently, Lin [17 determined the mod 3 cohomology of a simply connected homotopy associative and homotopy commutative mod 3 finite $H$-space. He has shown that the mod 3 cohomology $H^{*}(X)$ is isomorphic to the mod 3 cohomology of a finite product of $S p(2)$ s as an algebra over the mod 3 Steenrod algebra. His result is generalized to the space level. In fact, we can show the following:

Proposition 3.1. If $X$ is a simply connected homotopy associative and homotopy commutative mod 3 finite $H$-space, then $X$ is homotopy equivalent to a finite product of $S p(2) s$.

If $X$ is a simply connected homotopy associative and homotopy commutative mod 3 finite $H$-space, then by a result of Lin [17, we can see that

$$
H^{*}(X)=\Lambda\left(u_{1}, \cdots, u_{m}, \mathcal{P}^{1}\left(u_{1}\right), \cdots, \mathcal{P}^{1}\left(u_{m}\right)\right)
$$

with $\operatorname{deg} u_{i}=3$ for $1 \leq i \leq m$. It is known that the mod 3 cohomology of $S p(2)$ is given by

$$
H^{*}(S p(2))=\Lambda\left(u, \mathcal{P}^{1}(u)\right)
$$

with $\operatorname{deg} u=3$. To show Proposition 3.1, we need the following lemma:

Lemma 3.2. There exists a map $f_{i}: S p(2) \rightarrow X$ with $f_{i}^{*}\left(u_{i}\right)=u$ for $1 \leq i \leq m$.

Proof. From the mod 3 cohomology $H^{*}(S p(2))$, we have that

$$
S p(2) \simeq S^{3} \cup_{\alpha} e^{7} \cup_{\beta} e^{10},
$$

where $\alpha \in \pi_{6}\left(S^{3}\right) \cong \mathbb{Z} / 3$ is the generator and $\beta: S^{9} \rightarrow S^{3} \cup_{\alpha} e^{7}$ denotes some attaching map. Since $X$ is 3 -torsion free, the generators $u_{i} \in H^{3}(X)$ are the mod 3 reductions of the integral classes, and so we see that the 3-adic cohomology of $X$ is given by $H^{3}\left(X ; \mathbb{Z}_{3}^{\wedge}\right)=\mathbb{Z}_{3}^{\wedge}\left\{u_{1}, \cdots, u_{m}\right\}$. Since $X$ is a mod 3 finite $H$-space, we can assume that $X$ has a finite number of cells in each dimension (see [1, Prop. 3.2]), and so the 3 -adic homology $H_{3}\left(X ; \mathbb{Z}_{3}^{\wedge}\right)$ is a finitely generated $\mathbb{Z}_{3}^{\wedge}$-module. From the universal coefficient theorem, we have that $\pi_{3}(X) \cong H_{3}\left(X ; \mathbb{Z}_{3}^{\wedge}\right) \cong\left(\mathbb{Z}_{3}^{\wedge}\right)^{m}$, and so there exists a map $g_{i}: S^{3} \rightarrow X$ such that $g_{i}^{*}\left(u_{i}\right)=u$ on the mod 3 cohomology for $1 \leq i \leq m$. If we consider the three-connected cover of $X$, then the mod 3 cohomology is given by

$$
H^{*}(X\langle 3\rangle) \cong \mathbb{Z} / 3\left[x_{1}, \cdots, x_{m}\right] \otimes \Lambda\left(z_{1}, \cdots, z_{m}, \mathcal{P}^{3}\left(z_{1}\right), \cdots, \mathcal{P}^{3}\left(z_{m}\right)\right)
$$

with $\operatorname{deg} x_{i}=18$ and $\operatorname{deg} z_{i}=7$ for $1 \leq i \leq m$, which implies that $\pi_{k}(X) \cong$ $\pi_{k}(X\langle 3\rangle)=0$ for $4 \leq k \leq 6$ and $\pi_{7}(X) \cong\left(\mathbb{Z}_{3}^{\wedge}\right)^{m}$. If we consider a cofibration

$$
S^{6} \stackrel{\alpha}{\longrightarrow} S^{3} \stackrel{\gamma}{\longrightarrow} S^{3} \cup_{\alpha} e^{7}
$$

then we have that $g_{i} \alpha \simeq *$ since $\left[g_{i} \alpha\right] \in \pi_{6}(X)=0$, and so there exists a map $h_{i}: S^{3} \cup_{\alpha} e^{7} \rightarrow X$ with $h_{i} \gamma \simeq g_{i}$.

By computing the mod 3 cohomology of the 7 -connected cover of $X$, we have that $\pi_{k}(X) \cong \pi_{k}(X\langle 7\rangle)=0$ for $k=8,9$ and $\pi_{10}(X) \cong(\mathbb{Z} / 3)^{m}$. If we consider the following cofibration:

$$
S^{9} \stackrel{\beta}{\longrightarrow} S^{3} \cup_{\alpha} e^{7} \stackrel{\delta}{\longrightarrow} S p(2)
$$

then $\left[h_{i} \beta\right] \in \pi_{9}(X)=0$, which means that $h_{i} \beta \simeq *$. Hence we have a map $f_{i}: S p(2) \rightarrow X$ with $f_{i} \delta \simeq h_{i}$. This completes the proof.

Now we prove Proposition 3.1 as follows: 
Proof of Proposition 3.1. By Lemma 3.2, there exist maps $f_{i}: S p(2) \rightarrow X$ such that $f_{i}^{*}\left(u_{i}\right)=u$ for $1 \leq i \leq m$. By using the $H$-structure of $X$, we have a map $f: S p(2)^{m} \rightarrow X$ which induces an isomorphism on the mod 3 cohomology. This completes the proof of Proposition 3.1.

By using Proposition 2.3, Proposition 3.1 and results of Broto and Crespo [5], [8], we can prove Theorem A as follows:

Proof of Theorem A. By results of Broto and Crespo [5, [8], there exists an $H_{-}$ fibration

$$
K \longrightarrow X \longrightarrow F
$$

where $F$ is a simply connected mod 3 finite $H$-space and $K=K(\mathbb{Z}, 2)^{m}$ for some $m \geq 0$. Since the mod 3 cohomology $H^{*}(F)$ is finite dimensional, by Miller [20] Thm. A], we have that $F$ is $B \mathbb{Z} / 3$-local. By Proposition 2.1 , the localization functor $L_{B \mathbb{Z} / 3}$ with respect to $B \mathbb{Z} / 3$ preserves the $H$-fibration (3.1), and so is $F_{B \mathbb{Z} / 3}$. It is known by [2, Remark 9.5] that $F_{B \mathbb{Z} / 3}(K)$ is contractible, and so we have $F_{B \mathbb{Z} / 3}(X) \simeq F$. By Proposition 2.3, $F$ is a homotopy associative and homotopy commutative $\bmod 3$ finite $H$-space, and so by Proposition 3.1, $F \simeq S p(2)^{n}$ for some $n \geq 0$.

By extending the fibration (3.1), we have an $H$-fibration

$$
X \longrightarrow S p(2)^{n} \stackrel{\phi}{\longrightarrow} K(\mathbb{Z}, 3)^{m} .
$$

We can write that

$$
H^{3}\left(K(\mathbb{Z}, 3)^{m} ; \mathbb{Z}_{3}^{\wedge}\right)=\mathbb{Z}_{3}^{\wedge}\left\{\eta_{1}, \cdots, \eta_{m}\right\}
$$

and

$$
H^{3}\left(S p(2)^{n} ; \mathbb{Z}_{3}^{\wedge}\right)=\mathbb{Z}_{3}^{\wedge}\left\{u_{1}, \cdots, u_{n}\right\}
$$

where $\eta_{i} \in H^{3}\left(K(\mathbb{Z}, 3) ; \mathbb{Z}_{3}^{\wedge}\right)$ for $1 \leq i \leq m$ and $u_{j} \in H^{3}\left(S p(2) ; \mathbb{Z}_{3}^{\wedge}\right)$ for $1 \leq j \leq n$ are generators. If we consider the induced homomorphism $\phi^{*}: H^{3}\left(K(\mathbb{Z}, 3)^{m} ; \mathbb{Z}_{3}^{\wedge}\right) \rightarrow$ $H^{3}\left(S p(2)^{n} ; \mathbb{Z}_{3}^{\wedge}\right)$, then there exist generators $\tilde{\eta}_{i} \in H^{3}\left(K(\mathbb{Z}, 3)^{m} ; \mathbb{Z}_{3}^{\wedge}\right)$ for $1 \leq i \leq m$ and $\tilde{u}_{j} \in H^{3}\left(S p(2)^{n} ; \mathbb{Z}_{3}^{\wedge}\right)$ for $1 \leq j \leq n$ such that

$$
\phi^{*}\left(\tilde{\eta}_{i}\right)= \begin{cases}\tilde{u}_{i}, & 1 \leq i \leq s \\ 3^{e_{i}} \tilde{u}_{i}, & s+1 \leq i \leq t \\ 0, & t+1 \leq i \leq m\end{cases}
$$

where $e_{i} \leq e_{i+1}$ for $s+1 \leq i \leq t-1$. By using Lemma 3.2, we can construct a homotopy equivalence $\xi: S p(2)^{n} \rightarrow S p(2)^{n}$ such that $\xi^{*}\left(\tilde{u}_{j}\right)=u_{j}$ for $1 \leq j \leq n$. If $\zeta: K(\mathbb{Z}, 3)^{m} \rightarrow K(\mathbb{Z}, 3)^{m}$ denotes the map defined by $\zeta^{*}\left(\tilde{\eta}_{i}\right)=\eta_{i}$ for $1 \leq i \leq m$, then we have the following homotopy commutative diagram of fibrations:

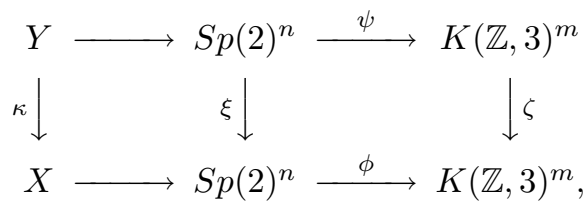

where

$$
Y=S p(2)\langle 3\rangle^{s} \times \prod_{i=s+1}^{t} S p(2)\left\langle 3 ; 3^{e_{i}}\right\rangle \times S p(2)^{n-t} \times K(\mathbb{Z}, 2)^{m-t}
$$


and $\psi: S p(2)^{n} \rightarrow K(\mathbb{Z}, 3)^{m}$ is the map defined by

$$
\psi^{*}\left(\eta_{i}\right)= \begin{cases}u_{i}, & 1 \leq i \leq s \\ 3^{e_{i}} u_{i}, & s+1 \leq i \leq t \\ 0, & t+1 \leq i \leq m\end{cases}
$$

From the five lemma, we have that $\kappa: Y \rightarrow X$ is also a homotopy equivalence. This completes the proof of Theorem A.

We can prove Corollary 1.2 as follows:

Proof of Corollary 1.2. If $\tilde{X}$ is the universal cover of $X$, then $\tilde{X}$ is also a homotopy associative and homotopy commutative mod $3 \mathrm{H}$-space, and there exists an $H_{-}$ fibration

$$
\tilde{X} \longrightarrow X \longrightarrow K\left(\pi_{1}(X), 1\right),
$$

where $K\left(\pi_{1}(X), 1\right)$ is a finite product of $K(\mathbb{Z}, 1)$ and $K\left(\mathbb{Z} / 3^{i}, 1\right)$ for $i \geq 1$. By a spectral sequence argument, we see that the mod 3 cohomology $H^{*}(\tilde{X})$ is also finitely generated as an algebra. By Theorem A, we have that

$$
\tilde{X} \simeq S p(2)\langle 3\rangle^{s} \times \prod_{i=s+1}^{t} S p(2)\left\langle 3 ; 3^{e_{i}}\right\rangle \times S p(2)^{n-t} \times K(\mathbb{Z}, 2)^{m-t},
$$

and so the mod 3 cohomology of $\tilde{X}$ is given by

$$
\begin{gathered}
H^{*}(\tilde{X}) \cong \mathbb{Z} / 3\left[x_{1}, \cdots, x_{s}, u_{s+1}, \cdots, u_{t}\right] \otimes \Lambda\left(z_{1}, \cdots, z_{s}, \mathcal{P}^{3}\left(z_{1}\right), \cdots, \mathcal{P}^{3}\left(z_{s}\right),\right. \\
\left.\beta_{e_{s+1}}\left(u_{s+1}\right), \cdots, \beta_{e_{t}}\left(u_{t}\right), \mathcal{P}^{1}\left(\beta_{e_{s+1}}\left(u_{s+1}\right)\right), \cdots, \mathcal{P}^{1}\left(\beta_{e_{t}}\left(u_{t}\right)\right)\right) \\
\otimes H^{*}\left(S p(2)^{n-t}\right) \otimes H^{*}\left(K(\mathbb{Z}, 2)^{m-t}\right)
\end{gathered}
$$

with $\operatorname{deg} x_{k}=18, \operatorname{deg} z_{k}=7$ for $1 \leq k \leq s$ and $\operatorname{deg} u_{i}=2$ for $s+1 \leq i \leq t$, where $\beta_{e_{i}}$ denotes the $e_{i}$-th Bockstein operation for $s+1 \leq i \leq t$.

By a result of Browder [6], we can use a spectral sequence for the $H$-fibration (3.2) to compute the mod 3 cohomology $H^{*}(X)$. If we assume that $d_{r}$ is the first non-trivial differential in the spectral sequence, then by the DHA lemma [12, p.14], the transgression $d_{r}: Q H^{r-1}(\tilde{X}) \rightarrow P H^{r}\left(K\left(\pi_{1}(X), 1\right)\right)$ is non-trivial, which implies that $r=2\left(3^{k}\right)$ for some $k \geq 1$. Since $Q H^{2\left(3^{k}\right)-1}(\tilde{X})=0$ by (3.3), we have $d_{r}=0$, and this causes a contradiction. Hence the spectral sequence collapses, and so we have that

$$
H^{*}(X) \cong H^{*}\left(K\left(\pi_{1}(X), 1\right)\right) \otimes H^{*}(\tilde{X}),
$$

which implies that the generators of $H^{*}(X)$ are concentrated in degrees 1,2,3,7,18 and 19. This completes the proof of Corollary 1.2.

\section{Proof of Theorem B}

This section is devoted to the proof of Theorem B. In $\S 2$ we have shown that the Dror Farjoun localization functor preserves the higher homotopy commutativity of loop spaces. By combining Proposition 2.5 with results of Broto and Crespo [5], [8], we can reduce the problem to the case of finite $C_{p}$-spaces.

In the case of finite $C_{p}$-spaces, McGibbon proved the following result: 
Theorem 4.1 ([19, Thm. 3]). Let $p$ be an odd prime. If $X$ is a simply connected finite $C_{p}$-space, then $X$ is contractible.

To prove Theorem B, we need the following lemma:

Lemma 4.2. If $X$ is a connected $C_{n}$-space, then the universal cover $\tilde{X}$ is a $C_{n}$ space so that the covering projection map $\omega: \tilde{X} \rightarrow X$ is a $C_{n}$-map.

Proof. We give an outline of the proof. Let $\mu: X \times X \rightarrow X$ denote the associative $H$-structure on $X$. By [21, Thm. II 4.2,4.3], there exists an associative $H$-structure $\tilde{\mu}: \tilde{X} \times \tilde{X} \rightarrow \tilde{X}$ on $\tilde{X}$ with $\omega \tilde{\mu}=\mu(\omega \times \omega)$.

Since $X$ is a $C_{n}$-space, there exist maps $G_{k}: X^{2(k+1)} \times I^{k} \rightarrow X$ for $0 \leq k<n$ satisfying the conditions (2.4) and (2.5). By using the covering lifting property (cf. 21, Lemma II 1.7]), we can construct a map $\tilde{G}_{k}: \tilde{X}^{2(k+1)} \times I^{k} \rightarrow \tilde{X}$ such that $\omega \tilde{G}_{k}=G_{k}\left(\omega^{2(k+1)} \times 1_{I^{k}}\right)$ for $0 \leq k<n$. From the uniqueness of the lifting, we can show that the maps $\tilde{G}_{k}: \tilde{X}^{2(k+1)} \times I^{k} \rightarrow \tilde{X}$ satisfies the conditions (2.4) and (2.5), and so $\tilde{X}$ is a $C_{n}$-space. This completes the proof.

Now we can prove Theorem B as follows:

Proof of Theorem B. If $\tilde{X}$ denotes the universal cover of $X$, then there exists an $H$-fibration

$$
\tilde{X} \longrightarrow X \longrightarrow K\left(\pi_{1}(X), 1\right),
$$

where $K\left(\pi_{1}(X), 1\right)$ is a finite product of Eilenberg-Mac Lane spaces $K(\mathbb{Z}, 1)$ and $K\left(\mathbb{Z} / p^{i}, 1\right)$ for $i \geq 1$. By Lemma 4.2 , we have that $\tilde{X}$ is a simply connected $C_{p}$-space, and by using a spectral sequence argument, we see that $H^{*}(\tilde{X})$ is also finitely generated as an algebra. By results of Broto and Crespo [5], [8], there exists an $H$-fibration

$$
K \longrightarrow \tilde{X} \longrightarrow F
$$

where $F$ is a simply connected $\bmod p$ finite $H$-space and $K$ is a finite product of $K(\mathbb{Z}, 2)$ s. By using the same arguments as in the proof of Theorem A, we have that $F_{B \mathbb{Z} / p}(\tilde{X}) \simeq F$, where $F_{B \mathbb{Z} / p}$ denotes the composite of the localization functor $L_{B \mathbb{Z} / p}$ with respect to $B \mathbb{Z} / p$ and the $p$-completion $(\mathbb{Z} / p)_{\infty}$. By Theorem 4.1 and Proposition 2.5, we see that $F$ is contractible, and so $\tilde{X} \simeq K$.

By a result of Browder [6], we can use a spectral sequence for the $H$-fibration (4.1) whose $E_{2}$-term is given by

$$
E_{2}^{*, *} \cong H^{*}\left(K\left(\pi_{1}(X), 1\right)\right) \otimes H^{*}(K) .
$$

From the DHA lemma [12, p.14], the spectral sequence collapses, and so we have that

$$
H^{*}(X) \cong H^{*}\left(K\left(\pi_{1}(X), 1\right)\right) \otimes H^{*}(K) .
$$

We can construct a map $\zeta: X \rightarrow K\left(\pi_{1}(X), 1\right) \times K$ which induces an isomorphism on the mod $p$ cohomology. Then $\zeta: X \rightarrow K\left(\pi_{1}(X), 1\right) \times K$ is a homotopy equivalence by a version of the Whitehead theorem (cf. [21, Cor. IV 1.6]), and so we have the required conclusion. This completes the proof of Theorem B.

Remark 4.3. In [13], we proved that if $X$ is a simply connected mod $p H$-space such that $H^{*}(\Omega X)$ is a finitely generated algebra, then $\Omega X$ is homotopy equivalent to a finite product of Eilenberg-Mac Lane spaces of dimensions 1 and 2 (see also 
14]). By combining a result of Aguadé-Smith [3] with the above argument, we have a simple proof of [13, Thm. A].

Lin [17] introduced the notion of an $A_{p-1}$-power $A_{p}$-space for an odd prime $p$. Let $X$ be an $A_{p}$-space in the sense of Stasheff [24]. $X$ is said to be an $A_{p-1}$-power $A_{p}$-space if the power map $\phi: X \rightarrow X$ defined by $\phi(x)=x^{\lambda}$ is an $A_{p-1}$-map, where $\lambda$ is a primitive $p$-th root of unity.

Let $B_{1}(p)$ denote the $S^{3}$-bundle over $S^{2 p+1}$ whose $\bmod p$ cohomology is given by

$$
H^{*}\left(B_{1}(p)\right) \cong \Lambda\left(u, \mathcal{P}^{1}(u)\right)
$$

with $\operatorname{deg} u=3$. It is known that for $p=3$ and $5, B_{1}(3)=S p(2)$ and $B_{1}(5)=G_{2}$, respectively (cf. [12, p.73]).

Lin [17, Thm. 5.4] has shown that the mod $p$ cohomology of a simply connected finite $A_{p-1}$-power $A_{p}$-space is isomorphic to the $\bmod p$ cohomology of a finite product of $B_{1}(p)$ s as an algebra over the $\bmod p$ Steenrod algebra. By using similar arguments to the proof of Proposition 3.1, we have the following:

Proposition 4.4. Let $p$ be an odd prime. If $X$ is a simply connected finite $A_{p-1^{-}}$ power $A_{p}$-space, then $X$ is homotopy equivalent to a finite product of $B_{1}(p) s$.

By Proposition 4.4, we can give a necessary condition for a simply connected $\bmod p$ loop space with finitely generated $\bmod p$ cohomology to be a $C_{p-1}$-space in the sense of Sugawara. Our result is as follows:

Theorem 4.5. Let $p$ be an odd prime. If $X$ is a simply connected $C_{p-1}-$ space such that the $\bmod p$ cohomology $H^{*}(X)$ is finitely generated as an algebra, then $X$ is homotopy equivalent to a finite product of $K(\mathbb{Z}, 2), B_{1}(p), B_{1}(p)\langle 3\rangle$ and $B_{1}(p)\left\langle 3 ; p^{i}\right\rangle$ for $i \geq 1$.

Here $B_{1}(p)\langle 3\rangle$ is the three-connected cover of $B_{1}(p)$, and $B_{1}(p)\left\langle 3 ; p^{i}\right\rangle$ denotes the homotopy fiber of the map of degree $p^{i}$ :

$$
\left[p^{i}\right]: B_{1}(p) \longrightarrow K(\mathbb{Z}, 3)
$$

for $i \geq 1$.

Remark 4.6. We recall that $X$ is a $C_{2}$-space if and only if $X$ is a homotopy commutative mod $p$ loop space. Since $B_{1}(3)=S p(2)$, the above theorem is obtained from Theorem A in the case of $p=3$.

We give an outline of the proof of Theorem 4.5.

Proof of Theorem 4.5. By results of Broto and Crespo [5], [8], there exists an $H_{-}$ fibration

$$
K \longrightarrow X \longrightarrow F
$$

where $F$ is a simply connected $\bmod p$ finite $H$-space and $K=K(\mathbb{Z}, 2)^{m}$ for some $m \geq 0$. By using the same arguments as in the proof of Theorem A, we have that $F_{B \mathbb{Z} / p}(X) \simeq F$, and so $F$ is a $C_{p-1}$-space by Proposition 2.5. From the definition of a $C_{p-1}$-space, the multiplication $\mu: F \times F \rightarrow F$ is an $A_{p-1}$-map. By using an induction, we see that the power map $\phi: F \rightarrow F$ is also an $A_{p-1}$-map, which implies that $F$ is an $A_{p-1}$-power $A_{p}$-space. By Proposition 4.4, $F$ is homotopy 
equivalent to a finite product of $B_{1}(p) \mathrm{s}$, and by extending the $H$-fibration (4.2), we have an $H$-fibration

$$
X \longrightarrow B_{1}(p)^{n} \longrightarrow K(\mathbb{Z}, 3)^{m} .
$$

By using similar arguments to the proof of Theorem A, we can show that

$$
X \simeq B_{1}(p)\langle 3\rangle^{s} \times \prod_{i=s+1}^{t} B_{1}(p)\left\langle 3 ; p^{e_{i}}\right\rangle \times B_{1}(p)^{n-t} \times K(\mathbb{Z}, 2)^{m-t},
$$

where $e_{i} \leq e_{i+1}$ for $s+1 \leq i \leq t-1$, and so we have the required conclusion. This completes the proof of Theorem 4.5.

Remark 4.7. Williams [26] introduced another kind of the higher homotopy commutativity of loop spaces. By a result of McGibbon [19, Prop. 6], if $X$ is a $C_{n}$-space in the sense of Sugawara, then $X$ is also a $C_{n}$-space in the sense of Williams. After we submitted this paper, the first author generalized Theorem B to the case of $C_{p}$-spaces in the sense of Williams. This result will be appearing in a forthcoming paper.

\section{REFERENCES}

[1] J. Aguadé, Cohomology algebras with two generators, Math. Z. 177 (1981), pp. 289-296. MR 82d:55003

[2] J. Aguadé, C. Broto, D. Notbohm, Homotopy classification of spaces with interesting cohomology and a conjecture of Cooke, Part I, Topology 33 (1994), pp. 455-492. MR 95i:55006

[3] J. Aguadé, L. Smith, On the mod $p$ torus theorem of John Hubbuck, Math. Z. 191 (1986), pp. 325-326. MR 87e:57044

[4] A. Bousfield, D. Kan, Homotopy limits, completions and localizations, Springer Lecture Notes in Math. 304 (1972). MR 51:1825

[5] C. Broto, J.A. Crespo, $H$-spaces with noetherian mod two cohomology algebra, Topology $\mathbf{3 8}$ (1999), pp. 353-386. MR 99i:55013

[6] W. Browder, The cohomology of covering spaces of $H$-spaces, Bull. Amer. Math. Soc. 65 (1959), pp. 140-141. MR 22:1891

[7] _ Homotopy commutative H-spaces, Ann. of Math. 75 (1962), pp. 283-311. MR 27:765

[8] J.A. Crespo, Structure of mod $p H$-spaces with finiteness conditions, Preprint.

[9] E. Dror Farjoun, Cellular spaces, null spaces and homotopy localization, Springer Lecture Notes in Math. 1622 (1996). MR 98f:55010

[10] K. Iriye, A. Kono, Mod p retracts of G-product spaces, Math. Z. 190 (1985), pp. 357-363. MR 88a:55017

[11] N. Iwase, A continuous localization and completion, Trans. Amer. Math. Soc. 320 (1990), pp. 77-90. MR 90k:55015

[12] R.M. Kane, The homology of Hopf spaces, North-Holland Math. Library 40 (1988). MR 90f:55018

[13] Y. Kawamoto, Loop spaces of H-spaces with finitely generated cohomology, Pacific J. Math. 190 (1999), pp. 311-328. MR 2000i:55027

[14] J.P. Lin, Loops of $H$-spaces with finitely generated cohomology rings, Topology Appl. 60 (1994), pp. 131-152. MR 95m:55022

[15] $\quad$ H-spaces with finiteness conditions, Handbook of Algebraic Topology, edited by I.M. James, North-Holland (1995), pp. 1095-1141. MR 97c:55017

[16] _ Finitely generated cohomology Hopf algebras and torsion, Pacific J. Math. 172 (1996), pp. 215-221. MR 97d:57049

[17] _ Homotopy commutativity, homotopy associativity and power spaces, J. Pure Appl. Algebra 134 (1999), pp. 133-162. MR 2000i:55045

[18] C. McGibbon, Homotopy commutativity in localized groups, Amer. J. Math. 106 (1984), pp. 665-687. MR 86a:55011 
[19] Higher forms of homotopy commutativity and finite loop spaces, Math. Z. 201 (1989), pp. 363-374. MR 90f:55019

[20] H.R. Miller, The Sullivan conjecture on maps from classifying spaces, Ann. of Math. 120 (1984), pp. 39-87.

[21] M. Mimura, H. Toda, Topology of Lie groups, I and II, Transl. Math. Monographs 91 (1991), Amer. Math. Soc. MR 92h:55001

[22] M. Slack, A classification theorem for homotopy commutative mod $2 \mathrm{H}$-spaces with finitely generated cohomology rings, Mem. Amer. Math. Soc. 92 (1991). MR 92k:55015

[23] On the cohomology of mod p homotopy commutative H-spaces, Math. Z. 211 (1992), pp. 121-127. MR 93h:55004

[24] J.D. Stasheff, Homotopy associativity of H-spaces I, II, Trans. Amer. Math. Soc. 108 (1963), pp. 275-292, 293-312. MR 28:1623

[25] M. Sugawara, On the homotopy commutativity of groups and loop spaces, Mem. College Sci. Univ. Kyoto Ser. A 33 (1960), pp. 257-269. MR 22:11394

[26] F.D. Williams, Higher homotopy-commutativity, Trans. Amer. Math. Soc. 139 (1969), pp. 191-206. MR 39:2163

Department of Mathematics, Faculty of Science, Hiroshima University, HigashiHiRoshima 739-8526, JAPAN

Current address: Department of Mathematics, National Defense Academy, Yokosuka 239-8686, Japan

E-mail address: yusuke@cc.nda.ac.jp

Department of Mathematics, University of California, San Diego, La Jolla, CaliFORNIA 92093-0112

E-mail address: jimlin@euclid.ucsd.edu 